\title{
Effects of Continuous kinaesthetic feedback based on tendon vibration on Motor Imagery $\mathrm{BCl}$ performance
}

\author{
M. Barsotti ${ }^{1}$, D. Leonardis ${ }^{1}$, N. Vanello ${ }^{2}$, M. Bergamasco ${ }^{1}$, A. Frisoli ${ }^{1}$ \\ ${ }^{1}$ PERCRO Laboratory, Scuola Superiore Sant'Anna, Pisa, Italy \\ 2 Department of Information Engineering / University of Pisa, Italy
}

\begin{abstract}
-
Background and objectives: Feedback plays a crucial role for using Brain Computer Interface (BCl) systems. This study proposes the use of vibration-evoked kinaesthetic illusions as part of a novel multisensory feedback for a Motor Imagery (MI) based $\mathrm{BCl}$ and investigates its contributions in terms of $\mathrm{BCl}$ performance and electroencephalographi (EEG) correlates.

Methods: Sixteen subjects performed two different right arm $\mathrm{MI}-\mathrm{BCl}$ sessions: with the visual feedback only and with both visual and vibration-evoked kinaesthetic feedback, conveyed by the stimulation of the biceps brachi tendon. In both conditions the sensory feedback was driven by the $\mathrm{MI}-\mathrm{BCl}$. The rich and more natural multisensory feedback was expected to facilitate the execution of $\mathrm{MI}$, and thus to improve the performance of the $\mathrm{BCl}$. The EEG correlates of the proposed feedback were also investigated with and without the performing of MI.

Results and Conclusion: The contribution of vibration-evoked kinaesthetic feedback led to statistically higher $\mathrm{BCl}$ performance (Anova, $F_{(1,14)}=18.1, p<.01$ ) and more stable EEG eventrelated-desynchronization (ERD). Obtained results suggest promising application of the proposed method in neuro-rehabilitation scenarios: the advantage of an improved usability could make the MI-BCls more applicable for those patients having difficulties in performing kinaesthetic imagery.
\end{abstract}

\section{INTRODUCTION}

Brain Computer Interface (BCI) is a growing technology that provides control over computer applications directly from the brain activity. BCI has found many applications ranging from basic communication and control for individuals with severe paralysis [1], to neuro-rehabilitation purposes [2]-[4].

In stroke rehabilitation, there are now sufficient evidences that non-invasive BCI may provide an advantage compared to traditional rehabilitation methods in patients with severe motor impairment [4]-[12]. In stroke patients motor recovery depends on the possibility to positively affect the neuroplastic changes associated with the brain lesion, and to perform motor training that maximize functional outcomes [13]. Hence, MI-BCI provides a valid substitute for active motor training as a mean to activate the motor network [14], [15], thus influencing motor recovery in a positive way [16].

Motor Imagery (MI), that is the mental rehearsal of a given action, is a challenging technique that often requires high concentration and long training phases to be reliably detected by an EEG based MI-BCI [17]. MI is based on the dynamics of brain oscillation described as event-related (de)synchronization (ERD,ERS) [18]. Unfortunately, there is a substantial percentage of subjects who are not able to achieve a good accuracy even after extended training sessions. Those subjects having difficulties performing MI, so called "BCI illiterate", could be patients that can potentially benefit most from this technology [19], [20]. In this context, the combination of MI with a congruent bio-feedback can provide a two-folded advantage: it generates a normal afferent-efferent feedback loop [13], useful for neuro-rehabilitation purposes, and improves consistency of MI features detected by BCI [21].

In [21] a hand exoskeleton was adopted to provide proprioceptive feedback by assisting opening and closing of the hand, showing how proprioceptive feedback (feeling and seeing hand movements) improved BCI performance significantly. Several works demonstrated that the use of vibrotactile and proprioceptive feedback in BCI enhanced the usability of the BCI systems [21]-[23]. As regards the vibrotactile feedback, both mechanical stimuli at the hand [22] and at the wrist [24] were proven to lead to an increase in classification accuracy when MI was performed in presence of stimulation.

Furthermore, the only selective sensation of vibrotactile stimulation, inducing a neural activity of the primary somatosensory cortex (S1), has been found to elicit an EEG desynchronization pattern similar to the one generated by the MI of the same portion of body. This fact could be explained both considering that the organization of the somatosensory map in S1 is similar to the somatotopic map of the motor homunculus in the precentral gyrus [25] and thus 
considering the important relationship between touch and movement. In fact, in an early study Goodwin and colleagues [26], discovered that specific vibration stimuli applied externally in proximity of muscle tendons (tendon vibration) evoke an illusory perception of movement. As a confirmation of this finding, it has been proven through Positron Emission Tomography (PET) technique [27], [28], that those specific vibrating stimuli, which are able to evoke a kinaesthetic illusion, induce the activation of motor cortex areas.

Since the tendon vibration is able to elicit sensations of limb movement without dependence on the users motor abilities [29], [30], it has shown interesting applications ranging from kinaesthetic illusion in virtual environment [31] to treatment of those affected by neuromuscular disorders [32], [33].

Yao and colleagues [34] proposed the use of kinesthesia illusion, evoked through tendon vibration, for calibrating an EEG based MI-BCI solely using the EEG sensation data acquired during the repeated presentation of the illusory stimulus. The subjects task was to perform left or right illusory sensation according to a given cue. Then, the subject was required to perform the correspondent MI task, integrating the sensory experience from the motor memory of the previous trial. At the end of each MI trial, subjects received a short vibration feedback (500 ms) according to the decoded brain activity.

\subsection{Objectives}

In this paper we propose that using the vibrationevoked illusory movement as a congruent continuous feedback during MI, can improve the MI-BCI classification accuracy. This substantially differs and extends the approach previously presented in [34], where tendon vibration, applied at the level of wrist, was used only for calibration of the BCI classification algorithm and for user's task guidance during the induced sensation task. We expect that providing a continuous tendon vibration feedback during the MI task, based on the decoded mental state of the subject, could significantly strengthen the observed event-related (de)synchronization (ERD,ERS), confirming our preliminary observations reported in [35].

The experiments conducted in the present study were designed to evaluate the incremental contribution of the visual plus vibration-evoked kinaesthetic feedback with respect to visual feedback alone in terms of BCI performance. We hypothesize an enhanced performance of MI resulting from the addition of the kinaesthetic feedback, attributable both to the improved congruency between MI and sensory feedback and to the involvement of more natural sensory afferents with respect to the only imagined motor task. The rest of the paper is structured as follows. The proposed system is described in Section 2, followed by the experimental description. Then, results are reported in Section 3 in terms of BCI performance and EEG analysis. Finally, discussion and conclusions are presented in Sections 4 and 5 respectively.

\section{Materials and Methods}

\subsection{Objectives}

The main goal of this study was to compare the MI-BCI performance under two different feedback conditions: the visual only feedback ( $\mathbf{V}$ session) and the visual plus the kinaesthetic feedback (VK session).

\subsection{Materials}

The experimental setup consisted of an apparatus for generating illusory movement by tendon vibration, a virtual representation of the subject's arm, and an EEG based MI-BCI.

Subjects seated on a comfortable chair in front of an LCD monitor. They wore the cap for EEG acquisition and their right arm was accurately positioned onto the device providing illusory kinaesthetic feedback. The elbow and the wrist were placed on comfortable supports, with the elbow angle at approximately 120 degrees as shown in Figure 1. Subjects were asked to keep the arm relaxed during each experimental session.

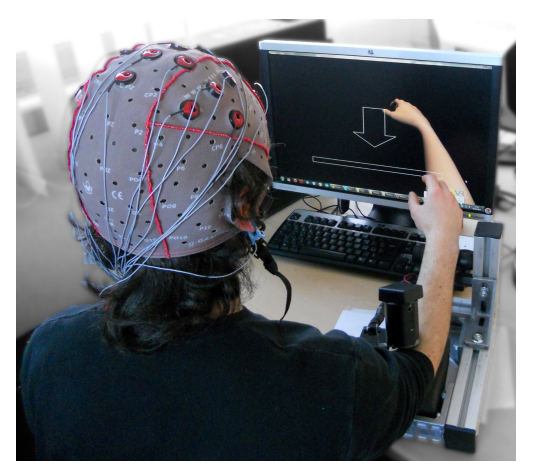

Fig. 1: Overview of the experimental setup

\subsubsection{The Tendon Vibration Stimulation Device}

The device used in this work, depicted in Figure 2, was designed and developed at the PERCRO Laboratory for eliciting illusory movements [35], and the illusory effect has been extensively investigated with the same device in both right- and left-handed individuals in [36]. The device was composed of an adjustable upper-limb support, and one actuator with adjustable position and orientation that can be finely positioned in proximity of muscle tendons. The tendon vibration was induced by means of an electromagnetic vibrator equipped with a skin tactor, applied externally to the biceps brachii tendon. Actuation was obtained by a linear voice-coil, allowing to accurately modulate the frequency patterns required for eliciting the motor 
illusion. The hemispherical plastic skin tactor, approximately $15 \mathrm{~mm}$ in diameter, was mounted at the tip of the moving shaft. Through the implemented device, it was possible to modulate independently the vibration frequency, the amplitude of the applied force and the pre-loading force generated by the tactor onto the skin. Figure 2 shows a general overview of the device with a representation of the operating phase (Figure 2(a)) and an inside view of the actuator (Figure 2(b)).

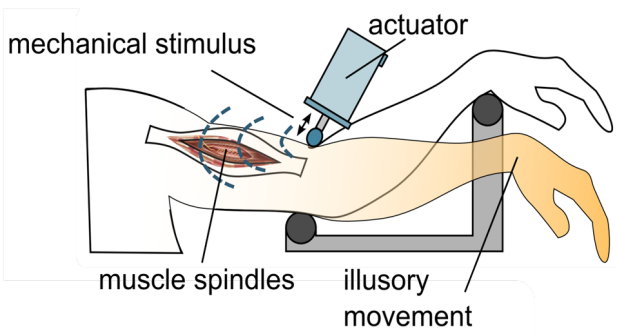

(a) Representation of the actuator position and the movement illusion

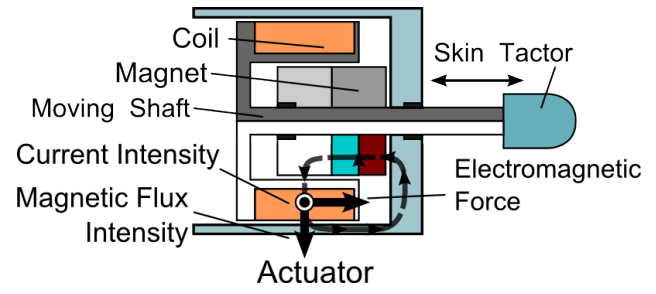

(b) Sketch of the inside view of the actuator

Fig. 2: Overview of the device used for illusory kinaesthetic stimulation.

\subsubsection{Visual Feedback}

Visual feedback of the performed mental task was provided to the subjects by means of a computer graphic avatar body. The scene, displayed onto an LCD monitor, was rendered from the point of view of the virtual avatar and the monitor was positioned in order to match subject's perspective. The movement executed by the virtual body was an extension of the right elbow, with a total displacement of 30 degrees from the resting position, congruent with the illusory movement that was expected by biceps brachii tendon vibration.

The monitor was also used for providing visual cues to the subject during the experimental session. In order to minimize eye movements, visual cues were displayed as wire-frame symbols (an arrow, a square or a cross) superimposed over the virtual scene, with just a minimal occlusion of the avatar body as can be seen in Figure 1.

\subsubsection{EEG recording}

The EEG based MI-BCI was based on the Event Related Desynchronization (ERD) phenomena in the $\mu$ $(8-13 \mathrm{~Hz})$ and $\beta(13-25 \mathrm{~Hz})$ bands. It is well known that these rhythmic components are involved in movement imagination tasks: in particular, an ERD is typically observed in the primary motor cortex, contralateral to the limb involved in the imaginary motor task [18]. The BCI was composed of an EEG acquisition system (active electrodes with gUSBamp amplifier from $g t e c^{\circledR}$ ) and a custom-made processing algorithm which executed in real-time the extraction and classification of EEG features, as shown in Figure 3 and explained in detail in the next section.

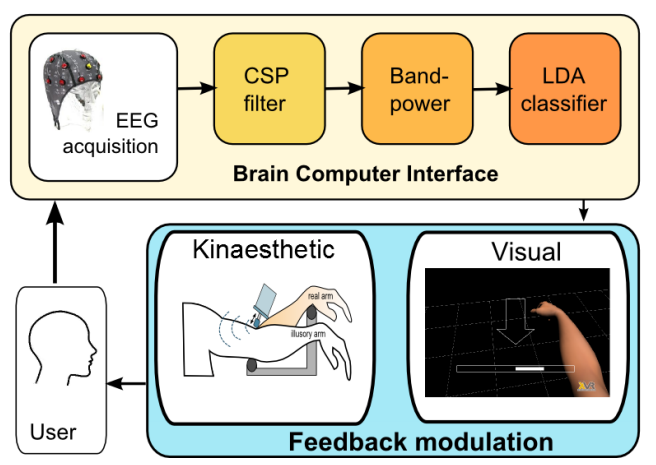

Fig. 3: Operation diagram of the experimental setup involving visual and kinaesthetic feedback driven by the $\mathrm{BCI}$

A pattern of thirteen active electrodes was placed over the sensorimotor cortex (Fc3, Fcz, Fc4, C5, C3, $\mathrm{C} 1, \mathrm{Cz}, \mathrm{C} 2, \mathrm{C} 4, \mathrm{C} 6, \mathrm{Cp} 3, \mathrm{Cpz}, \mathrm{Cp} 4)$ and two additional electrodes were placed in $\mathrm{Fp} 1$ and $\mathrm{Fp} 2$ for the eye-blink artifact removal in the post processing analysis. The ground electrode was placed in Afz and all channels were referenced to the right earlobe. Signals were pre-filtered in the $2-30 \mathrm{~Hz}$ frequency band (bank filter embedded in the g.USBamp) and then digitally converted with sample frequency of 256 $\mathrm{Hz}$ and resolution of 24 bits.

\subsection{Methods}

\subsubsection{Participants and Inclusion criteria}

Sixteen healthy right-handed subjects $(6 \mathrm{f} .10 \mathrm{~m}$. , aged 23-32) participated to the study. The experiments were conducted in accordance with the WMA Declaration of Helsinki and all subjects provided written consent to participate. All but two subjects had no previous experience with MI-BCI. Subjects' capability of perceiving the motor illusion was assessed by applying a vibro-mechanical stimulus (frequency $80 \mathrm{~Hz}$, duration $8 \mathrm{~s}$ ) in proximity of the insertion of the biceps brachii tendon at the elbow joint. Then subjects were asked to qualitatively report the vividness of the perceived illusion of movement and the position of the skin tactor was adjusted up to elicit a vivid illusion of movement. The procedure lasted up to a maximum of 15 minutes and Subjects who did not feel any movement illusion, or were not able to relax muscles 
were not included in the study (1 subject out of 17 participants). No subjects were discarded depending on their ability of performing MI.

\subsubsection{Experimental Design and Statistical Analysis}

A 2x2 mixed factorial design was adopted with factors the type of bio-feedback received during MI-BCI (V vs. VK feedback, within-subjects factor), and the type of received training (V- vs. VK-training, betweensubjects factor). A 2-way mixed Anova was conducted for evaluating the BCI performance homogeneity of variances was assessed with the Levene's Test. The scheme of the experimental design is shown in Figure 4.

For disambiguating the effect of the type of feedback received during the training session on BCI performance, participants were divided in two groups of 8 subjects each: one group performed the training session receiving the Visual only feedback (V-training group) and the other group both the Visual and Kinaesthetic feedback (VK-training group).

Each subject was then randomly assigned to $\mathrm{V} / \mathrm{VK}$ or $\mathrm{VK} / \mathrm{V}$ sequence of validation sessions, for avoiding the effect of fatigue and practice.

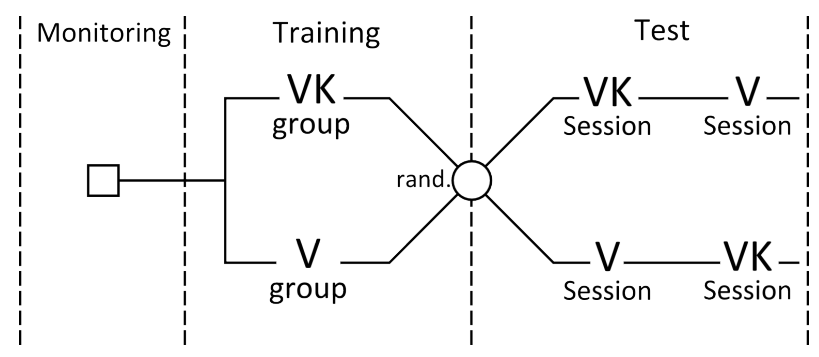

Fig. 4: Scheme of the experimental design.

\subsubsection{Experimental procedure}

The experimental procedure consisted of four sessions sharing the same trial structure depicted in Figure 5.

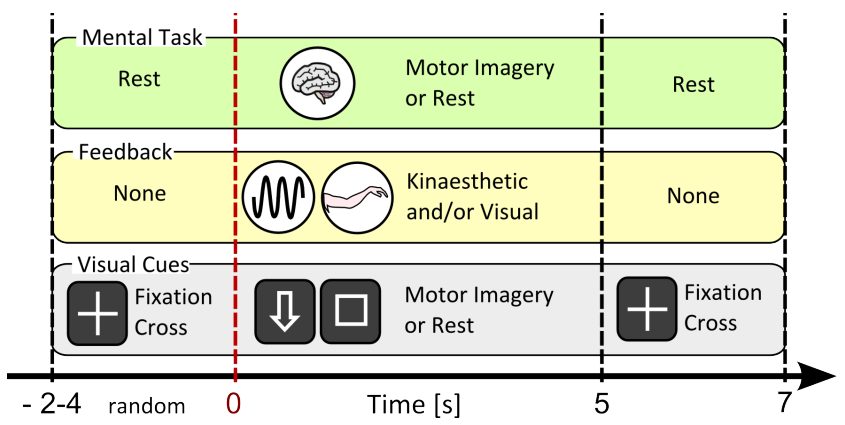

Fig. 5: Graphic representation of the trial structure

Each trial lasted for 7 seconds and was spaced from the next by a random duration interval lasting from 2 to 4 seconds allowing subject to relax concentration.
Each task was triggered by means of visual cues superimposed to the virtual arm in the LCD screen. Cues were in the form of an arrow pointing downwards for the "move" class, and a square for the "rest" class.

The four session are separately described below in details, and summarized in Table 1.

In the monitoring session (M), kinaesthetic and visual stimulation of the right arm movement was provided to the subjects for 20 trials. In this session, which was presented to each subject at the beginning of the experimental procedure, subjects were asked to passively perceive the stimuli without performing any mental task. The $\mathrm{M}$ session was conducted for collecting EEG data related to sensation of the multisensory feedback without the MI activity.

In the following experimental sessions subjects were repeatedly asked either to perform an imaginary movement of the right arm ("move") or to hold a resting mental state ("rest"). Each session was composed of a randomly sorted sequence of 40 trials, 20 for each mental task.

\begin{tabular}{|c|c|c|c|c|c|c|}
\hline Session & Name & $\begin{array}{c}\text { Mental } \\
\text { Task }\end{array}$ & Feedback & \begin{tabular}{|c|}
$\#$ \\
Subjects \\
\end{tabular} & \begin{tabular}{|c|}
$\mathrm{BCl}$ \\
driven
\end{tabular} & $\begin{array}{c}\# \\
\text { Trials } \\
\end{array}$ \\
\hline Monitoring & M & Rest & $\begin{array}{c}\text { Visual and } \\
\text { Kinaesthetic }\end{array}$ & 16 & No & 20 \\
\hline \multirow[b]{2}{*}{ Training } & \multirow[b]{2}{*}{$\mathrm{T}$} & \multirow[b]{2}{*}{ Rest + MI } & $\begin{array}{c}\text { Visual } \\
\text { (V-training) }\end{array}$ & 8 & \multirow[b]{2}{*}{ No } & \multirow[b]{2}{*}{$20+20$} \\
\hline & & & $\begin{array}{l}\text { Visual and } \\
\text { Kinaesthetic } \\
\text { (VK-training) }\end{array}$ & 8 & & \\
\hline \multirow[b]{2}{*}{ Test } & V & Rest + MI & Visual & 16 & Yes & $20+20$ \\
\hline & VK & Rest + MI & $\begin{array}{c}\text { Visual and } \\
\text { Kinaesthetic }\end{array}$ & 16 & Yes & $20+20$ \\
\hline
\end{tabular}

TABLE 1: Sessions description. BCI-driven feedback were provided according to the $\mathrm{BCI}$ output, whereas in the $\mathrm{M}$ and $\mathrm{T}$ sessions feedback were provided according to each trial. The presentation order of test sessions (V and VK) was randomized over subjects.

In the BCI training session (T), the only one which differs among the two groups, subjects were asked to actively perform motor imagery of the right arm while receiving visual only feedback (V-training group) or both kinaesthetic and visual feedback (VK-training group) congruently with the task. More in detail, the vibrational stimulus and the avatar movement were presented to the subjects only in the "move" trials, whereas during "rest" trials no kinaesthetic feedback and visualization of a still virtual arm were provided.

At the end of the $\mathrm{T}$ session, the recorded EEG data were processed for determining the BCI parameters for driving online the multisensory feedback in the next two sessions (see following Sections for further details on the BCI algorithm).

The visual-kinaesthetic condition (VK) was performed with both visual and vibration-evoked kinaesthetic feedback active, whereas in the visual condition (V) the visual feedback only was active. In order to 
minimize learning effects, the presentation order of the $\mathrm{V}$ and $\mathrm{VK}$ sessions was randomized over subjects.

While in the $\mathrm{M}$ and $\mathrm{T}$ sessions feedback was presented to the subjects accordingly to the current trials, in the subsequent $\mathrm{V}$ and VK sessions feedback stimuli were driven by the real-time BCI output with CSP and SVM parameters calculated in T session. In particular, when the feedback was BCI-driven, the BCI detection of the "move" class caused the virtual arm to move and the kinaesthetic stimulation to activate. On the other hand, during the detection of the "rest" class, the virtual arm was stopped and the kinaesthetic stimulation deactivated. In all conditions, the virtual arm was smoothly recovered to the starting position during the interval period between two subsequent tasks.

\subsubsection{The $\mathrm{BCl}$ algorithm}

Two mental states were intended to be classified, associated respectively to the right arm motor imagery ("move") and to the resting state ("rest"). The first stage of the BCI processing algorithm was a linear spatial filter based on the well-known Common Spatial Pattern (CSP) algorithm [37]. The CSP algorithm implements a supervised spatial filtering method for two-class discrimination problems, finding the directions that maximize variance for one condition and at the same time minimize variance for the other condition. Since variance of band-pass filtered signals is equal to band-power, CSP filters are well suited for discriminating mental states characterized by spectral perturbations effects and they have been widely applied to ERD detection in motor imagery based BCIs [12], [38]-[40]. Moreover, since the CSP is a data driven approach, it increases adaptability to subjectspecific features.

Mathematically, CSP is realized by simultaneous diagonalization of the covariance matrices of data acquired in the two conditions to be classified. Given $X$ a $N \times M$ data matrix with $N$ the number of channels and $M$ the number of time points, the CSP algorithm determines a spatial filter matrix $W$ such that $Z=W X$ are the projected data, and the row of $W$, called here $w_{i}$, are the spatial filters. The weights of $W$ are determined by finding the solution of the following minimization problem:

$$
\min _{w_{i}}\left(\sum_{i=1}^{m} w_{i} C_{2} w_{i}^{T}+\sum_{i=m+1}^{2 m} w_{i} C_{1} w_{i}^{T}\right)
$$

subject to:

$$
\begin{array}{ll}
w_{i}\left(C_{1}+C_{2}\right) w_{i}^{T}=1 & i=1,2, \ldots, 2 m \\
w_{i}\left(C_{1}+C_{2}\right) w_{j}^{T}=0 & i, j=1,2, \ldots, 2 m \quad i \neq j
\end{array}
$$

where $C_{1}$ and $C_{2}$ are the covariance matrices of data belonging to the two classes. The projection obtained using the first row of $W$ allows achieving the highest, normalized variance for class 1 , while projection using the last row of $W$ allows obtaining the lowest, normalized variance for class 2 .

In the online signal processing, EEG signals were projected using the first and last CSP components. Then, the 8-24 Hz band-power was computed for the two projected channels, using a 1 second moving time window, shifted by one sample at each execution step ( $256 \mathrm{~Hz}$ frequency of execution). The two band-power values were log-scaled and used as a two-dimensional feature for classification of the brain activity.

A Support Vector Machine (SVM) linear classifier was used to compute the distance of the extracted features from the classification threshold between the "move" and "rest" classes. The output of the linear classifier was low pass filtered ( $0.5 \mathrm{~s}$ time constant) and it represented the continuous BCI output (positive and negative values are related to the "move" or "rest" class, respectively). The weights of the spatial filter (CSP) and of the linear classifier (SVM) were trained offline using data acquired during the $\mathrm{T}$ session, which differed between $\mathrm{V}$-training group and VK-training group, and used for the V and VK sessions.

Considering the whole signal processing, the BCI output was approximately delayed of about 1 second with respect to the current mental activity of the subjects and the feedback was modulated in real-time by the output of the BCI.

\subsubsection{Data analysis}

The $\mathrm{BCI}$ performance during the $\mathrm{V}$ and $\mathrm{VK}$ conditions have been compared both online (actual BCI performance) and offline (post-processed EEG data and simulated BCI output). In particular, the online $\mathrm{BCI}$ performance consisted in the real-time $\mathrm{BCI}$ output obtained with $\mathrm{BCI}$ parameters tuned on the $\mathrm{T}$ session. For the off-line BCI performance, the EEG data were cleaned from eye-blink and muscular artifacts, then, the BCI output was simulated using BCI parameters trained with EEG data recorded in the same session. In order to compute the correct classification rate the BCI output was transformed accordingly to each trial class in a binary signal assuming values equal to zero and one for wrong and correct classification respectively. The correct classification rate was then calculated in the time range between 1.5 and 3.5 seconds of each trial and it was used in the following statistical analysis. All the offline EEG data processing, such as epoching, denoising and time-frequency transformation, have been conducted using EEGLAB software [41]. Time-frequency analysis, such as eventrelated spectral perturbation (ERSP) and its variants event-related de/synchronization (ERD/ERS), were computed using the full-epoch length single-trial correction method, in conjunction with the baseline permutation statistical method for inference testing [42]. 
We used a total of 500 permutations at each frequency for assessing significance with a $p$-value of 0.05 . Since the baseline normalization is performed assuming the additive ERSP model, the results are shown as $\mathrm{z}^{-}$ scores. The time interval of 1.5 seconds before the onset of the visual cue was chosen as the time baseline for normalization.

The time-frequency decomposition was performed using a Morlet wavelet with a moving window of 1 second. The number of cycles in each Morlet wavelet increased linearly with frequency using 3 cycles at lowest frequency to 30 at highest estimating 27 linearspaced frequencies from $3.0 \mathrm{~Hz}$ to $30.0 \mathrm{~Hz}$.

\section{Results}

The following section shows a comparative analysis between $\mathrm{V}$ and VK sessions in terms of BCI performance and the EEG data analysis related to the four experimental sessions.

\section{1 $\mathrm{BCl}$ performance}

The time-continuous correct classification rate of the $\mathrm{BCI}$ output, averaged over trials and subjects is shown in Figure 6. As expected, the classification rate at the beginning of each trial is about the chance $(50 \pm 15 \%$ for a 2-class discrimination paradigm with 20 trials per class [43]), then it increases with a delay of about 1 second. Correct rate obtained by each subject in the two feedback conditions are reported in Figure 7.
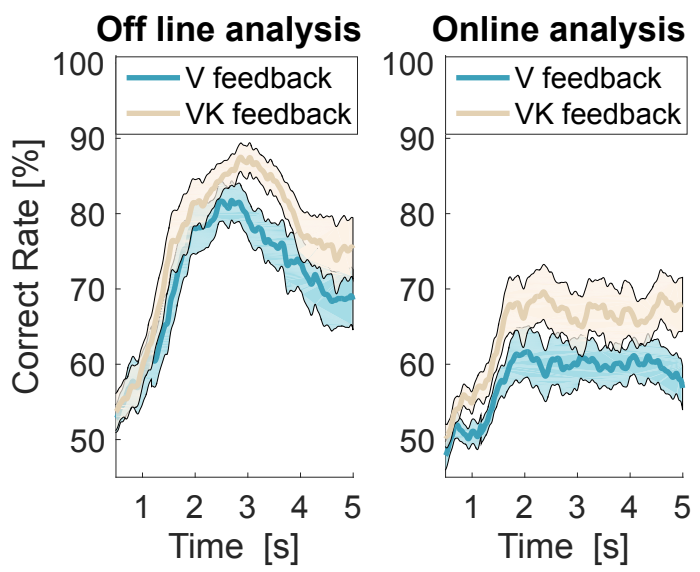

Fig. 6: Comparison of the classification performance over time averaged over subjects and trials.

Marginal means for the two feedback conditions and for the two training conditions are reported in Figure 8 and in Table 2.

The Anova results highlighted the same significant effects both for the online and offline performance. A significant higher performance was found in the VK sessions, (effect of Feedback, online: $F_{(1,14)}=18.1$, $p<.01, \eta_{p}^{2}=0.56$; offline: $F_{(1,14)}=21.5, p<.01$, $\eta_{p}^{2}=0.61$ ) while no interaction effect was found

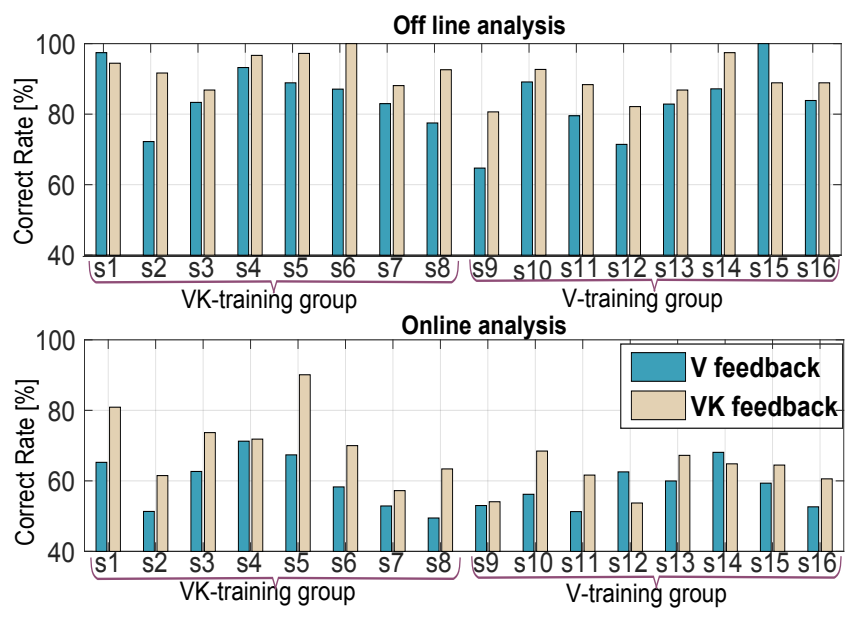

Fig. 7: BCI-Performance for each subject (see Sec 2.3.5 for details about the correct rate computation).

between Feedback and Training (online: $F_{(1,14)}=1.79$, $p=0.20$; offline: $\left.F_{(1,14)}=0.12, p=0.74\right)$. The effect of Training was also non-significant (online: $F_{(1,14)}=$ $3.35, p=0.09$; offline: $\left.F_{(1,14)}=2.21, p=0.16\right)$. This means that the higher performance achieved in the VK session did not depend on the type of training performed.
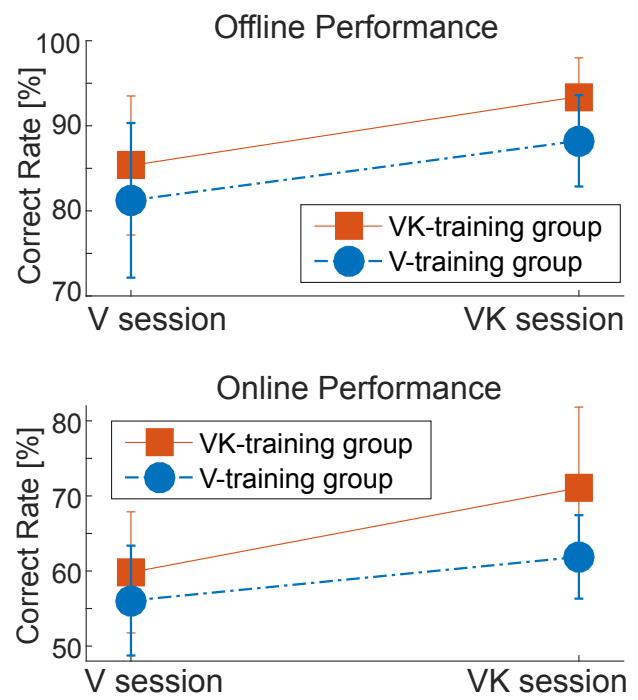

Fig. 8: BCI correct rate obtained with the Visual feedback (V session) and with the Visual and Kinaesthetic feedback (VK session) averaged over subjects.

Bar graphs in Figure 9 show the averaged correct classification rate obtained in the V and VK sessions for the two groups. The significant differences are reported considering the Anova results with and multivariate tests.

\subsection{EEG data analysis}

In this section the analysis of EEG data in terms of sensory-motor rhythms modulation is reported. 

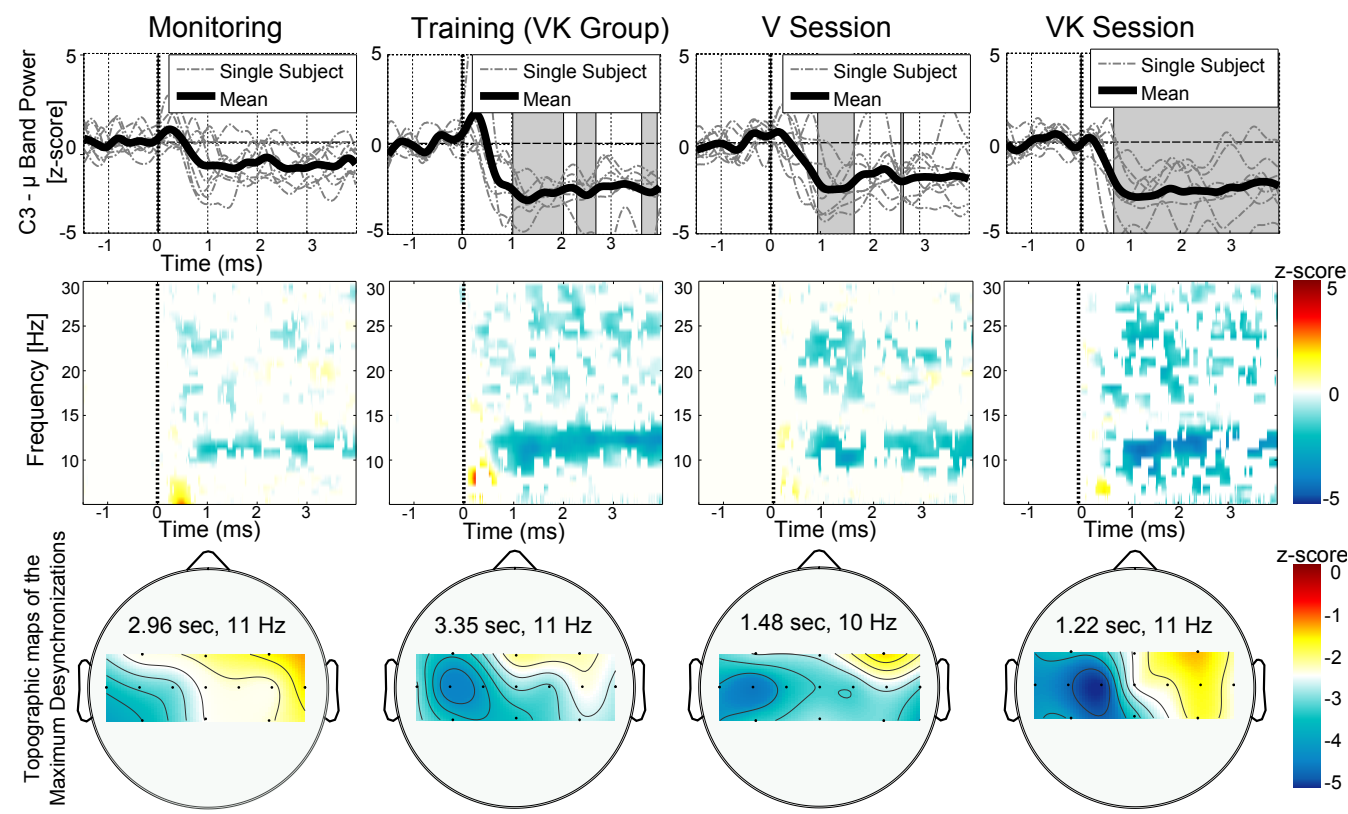

Fig. 10: Comparison of the EEG correlates of the VK-training group in the four experimental conditions. First row: power spectra of the 8-13 Hz band, $\mathrm{C} 3$ electrode representing right hand area in the primary motor cortex; Second Row: Time-frequency plot averaged over "move" trials, subjects and electrodes (only significant values are colored); Third Row: scalp maps related to the maximum desynchronization of the corresponding timefrequency plots. In order to improve spatial resolutio of the scalp maps the colormap in the third row assume only negative values (ERD).

\begin{tabular}{|c|c|c|r|}
\hline Online & V session & VK session & Mean \\
\hline V-training group & $56.08 \pm 7.32$ & $61.88 \pm 5.56$ & $58.97 \pm 6.44$ \\
\hline VK-training group & $59.82 \pm 8.06$ & $71.80 \pm 10.75$ & $65.45 \pm 9.41$ \\
\hline Mean & $57.94 \pm 7.69$ & $66.48 \pm 8.16$ & $62.21 \pm 7.92$ \\
\hline \hline Off-line & V session & VK session & Mean \\
\hline V-training group & $81.24 \pm 9.09$ & $88.24 \pm 5.37$ & $84.74 \pm 7.23$ \\
\hline VK-training group & $85.33 \pm 8.17$ & $93.44 \pm 4.55$ & $89.39 \pm 6.36$ \\
\hline Mean & $83.29 \pm 8.63$ & $90.84 \pm 4.96$ & $87.06 \pm 6.79$ \\
\hline
\end{tabular}

TABLE 2: Averaged correct rate performance.
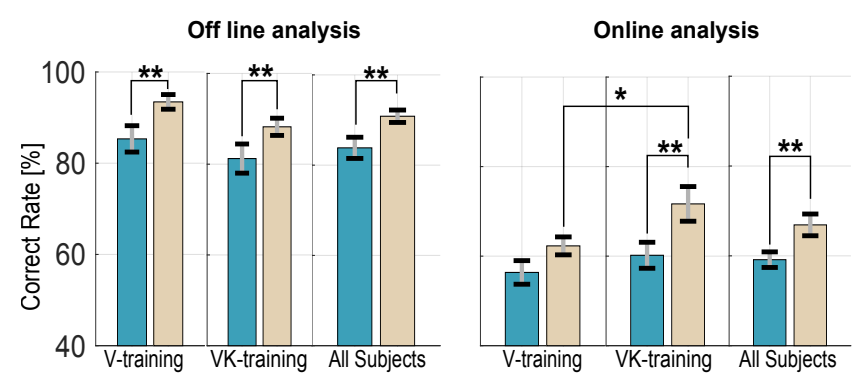

Fig. 9: BCI performance averaged over subjects. $\left({ }^{*} p<\right.$ $\left.0.05,{ }^{* *} p<0.01\right)$

Results for subjects belonging to the two experimental groups were similar and, for the sake of space, only the analysis of the VK training group is reported. Data belonging to "move" trials, in which the mental task was to perform MI, have been compared between all the experimental sessions and results are reported in
Figure 10 (see Section 2.3.3 for a detailed description of each session). The vertical dotted lines at the time zero of graphs in the first and second rows, mark the beginning of "move" trials, corresponding to the presentation of the visual cue. The first row shows the $\mu$-band $(8-13 \mathrm{~Hz})$ ERD at the electrode position C3 (overlying the cerebral motor cortex area representing the right hand according to the somatotopic map). The dotted gray lines indicate the time courses of the $\mu$ bandpower averaged for each subject, while the black solid line represents the average over the subjects. Significant regions (permutation statistic, $p<0.05$ ) are highlighted with a gray background area. No significant ERD was found in the M condition. Conversely, a significant ERD starting after about 1 second from the stimulus presentation appears in all the other experimental conditions. While in the $\mathrm{V}$ condition there are two significant regions lasting together about 1 second, in the VK condition appears a wider significant region starting about 1 second after the stimulus presentation and lasting about $3 \mathrm{~s}$. Also in the $\mathrm{T}$ session, in which the same multisensory feedback of the VK session was provided (VK-training group), it emerges a cumulative significant region lasting about two seconds. Time-frequency plots in the second row of Figure 10 show only the significant ERD (blue) and ERS (red) averaged over electrodes, "move" trials and subjects. Values are considered significant whereas there is at least one electrode with a significant value in all subjects. The scalp maps in the third row show 
the spatial distribution of the maximum negative peak (maximum ERD), corresponding to a point in the relative time-frequency plot (coordinates in the time and frequency domain are reported over each scalp plot). All topographic maps resulted centered on the contra-lateral sensory motor cortex (left hemisphere). In particular, the deepest ERD, focused on the C3 electrode position, can be noticed for the VK condition whithin the $\mu$ band $(11 \mathrm{~Hz})$ after $1.22 \mathrm{~s}$ from the presentation of the stimulus.

\section{Discussion}

In this work we proposed the use of the kinaesthetic illusion based on the biceps brachii tendon vibration as a continuous feedback for a MI-BCI, and we investigated its contribution in terms of $\mathrm{BCI}$ performance and EEG correlates. In order to obtain a multisensory afferent signal congruent with the MI task, the kinaesthetic illusion was provided to the subjects coupled with the visual feedback of a virtual arm. The substantial novelty introduced by this work relies on the use of continuous kinaesthetic illusory feedback provided accordingly to the subjects' decoded mental state, and thus simultaneously to the performing of MI.

In order to improve training of the BCI parameters, we designed a training session in which subjects received sensory stimuli congruently with the performing of MI. This training paradigm led to the twofold advantage of assuring a consistent ERD pattern detectable by the BCI system (as can be observed by the time-frequency graph in Figure 10) and facilitating subjects in performing MI. In this way, we were able to enroll BCI naive subjects without evaluating their capability of performing MI, and to avoid a prolonged training phase. In order to overcome the issue of a bias introduced by the feedback similarity between the training session and the test session, subjects were divided in two groups which differed by the type of feedback received during the training sessions: visual only (V-training group) and visual plus kinaesthetic (VK-training group). In the test sessions (V and VK sessions), all subjects were able to use the MI-BCI system with a reasonable classification accuracy as reported in Figures 9 and 6. The relatively low correct rate achieved in the online session (about 65\%), and the $20 \%$ discrepancy between the online and off-line analysis, could be explained by the fact that the online feedback was evaluated without any post-processing. This choice has been done for reporting data as closest as possible to the actual use of the BCI system.

BCI performance have been evaluated through a 2way mixed anova with respect to the type of feedback received in test and training sessions. Performance achieved by the subjects in the VK-session was significanlty better than V-session and no interaction effect was found between training and feedback, demonstrating that the better performance achieved in the
VK-session did not depend on the type of feedback received. Furthermore, although not statistically significant, as suggested by [34], the slightly better performance achieved by the VK-training group can be addressed to the more detectable EEG features the BCI system was trained on.

From the EEG data analysis conducted on the VKtraining group, it emerged that the proposed multisensory stimulation in absence of MI ( $\mathrm{M}$ condition) was sufficient to elicit alone a noticeable $\mu$-band ERD (Figure 10, first row). This result is in line with the recent work of Yao and colleagues [34] in which they demonstrated that a MI-BCI could be trained using a model calibrated solely through kinesthesia stimulation of wrist extensor tendon. In terms of EEG correlates, the vibration-evoked kinaesthetic feedback contributed to a longer and deeper EEG desynchronization during motor imagery than in presence of the visual feedback only, and thus it could be claimed that the effect of the proposed afferent stimulation increases the reliability of the MI ERD features (Figure 10). In fact, as shown in the time-frequency graphs and the correspondent topographic maps in Figure 10 , the analysis conducted with data acquired during the performing of MI (T, V and VK sessions), resulted in a more pronounced ERD focused on the contralateral area of the motor cortex. The most focused and contrasted contra-lateral pattern, localized on the scalp site proximal to the representation of the right hand in the primary sensory motor cortex, was found for the VK session where the multisensory feedback was provided congruently to the decoded MI. This fact could be linked to the activation of the sensorymotor network due to both the performing of MI and the illusory sensation [44]-[46].

Since the MI-BCI is based on the detection of ERD occurring during motor imagery, it could be expected that the addition of vibration-evoked kinaesthetic feedback might have influenced the BCI performance by simply biasing depth and stability of ERD. However, two elements have to be considered: firstly, the correct ratio of the BCI is the balanced outcome of correct detection of both rest and movement classes. Secondly, feedback in V and VK condition was driven by the BCI output classification independently of the presented mental task (hence in case of wrong classification, feedback could have been active also during "rest" trials). Therefore, the higher correct ratio for the VK condition suggests that multisensory feedback facilitated the use of the MI-BCI, and did not simply introduce a bias due to EEG correlates of the kinaesthetic stimulation. Yet, due to the relatively short duration of trials $(8 \mathrm{sec}-$ onds), the present study does not investigate the possible difficulty in mentally switching from MI to resting state once the feedback has been activated. This experimental structure reflects the common BCI application in neuro-rehabilitation studies, in which 
a sequence of motor tasks is presented to the patients. Facilitation in the use of the MI-BCI might be generated by the improved coherence of the afferent pathways with the mental task while performing motor imagery in presence of multisensory feedback. Obtained results showed this approach as promising for being introduced in neuro-rehabilitation scenarios, where the connection between the mental action and the perceived feedback constitutes a critical factor [2], [11], [13]. Moreover, due to the purely "virtual" nature of the proposed multisensory feedback, the presented method could also be applied with those patients who are severely impaired and present highly impeded motor capabilities (in particular at the early stage of the rehabilitation). Finally, since the proposed method fits particularly well with virtual application, it offers a high flexibility and easy parametrization of the scenario to the specific needs of individual patients.

\section{Conclusions}

The study investigated the contribution of kinaesthetic illusions evoked by tendon vibration as part of a multisensory feedback for MI-BCI. When driven by MI, the proposed afferent multisensory signal aimed to be a congruent and more natural bio-feedback the brain receives as a consequence of the efferent motor intention. Thus, improvements in performing motor imagery and consequently in BCI performance were expected. From experiments conducted in this work, it resulted that the congruent multisensory feedback (visual, through a virtual representation of the body, and kinaesthetic, through vibration-evoked motor illusions), significantly increased the MI-BCI performance with respect to the use of the visual feedback alone. Regarding the EEG correlates, it has been found that the measured ERD was deeper and more stable in presence of the multisensory feedback than in presence of visual feedback alone. Interesting applications are envisaged in the neuro-rehabilitation scenario. The improved voluntary control of MI due to the congruent multisensory feedback can be exploited both for a more effective motor network training, and for increasing MI-BCI usability for those subjects having difficulties performing kinaesthetic imagery. An aspect emerged from the study that require further investigation is whether the activation of the multisensory feedback may hinder the recovery of the rest mental state. In the present work this aspect did not emerged since, as in the most of rehabilitative BCI systems, BCI was operated in a structured sequence of short lasting trials. Instead, in an assistive BCI system, in which the BCI is fully self-paced without any trial structure, this could become a crucial point. Regarding neuro-rehabilitation applications, the proposed method allows to exercise MI with congruent multisensory feedback by generating movement perception in a completely virtual form - through the virtual body representation and vibration-evoked kinaesthetic illusions. Since no overt movements are required, this approach offers the possibility to include patients with limited motor capabilities, and provides the flexibility of a purely virtual rehabilitation scenario, parametrized to the specific needs of the patient.

\section{ACKNOWLEDGMENTS}

This work has been partially funded from the EU Horizon2020 project nr.644839 ICT-23-2014 CENTAURO and by RONDA project funded by Regione Toscana, Italy within the FAS "Salute" 2014 program.

\section{REFERENCES}

[1] N. Birbaumer, G. Gallegos-Ayala, M. Wildgruber, S. Silvoni, and S. R. Soekadar, "Direct brain control and communication in paralysis," Brain topography, vol. 27, no. 1, pp. 4-11, 2014.

[2] S. R. Soekadar, S. Silvoni, L. G. Cohen, and N. Birbaumer, "Brain-machine interfaces in stroke neurorehabilitation," in Clinical Systems Neuroscience. Springer, 2015, pp. 3-14.

[3] J. d. R. Millán, R. Rupp, G. R. Müller-Putz, R. Murray-Smith, C. Giugliemma, M. Tangermann, C. Vidaurre, F. Cincotti, A. Kübler, and R. Leeb, "Combining brain-computer interfaces and assistive technologies: state-of-the-art and challenges," Frontiers in neuroscience, vol. 4, 2010.

[4] M. Barsotti, D. Leonardis, C. Loconsole, M. Solazzi, E. Sotgiu, C. Procopio, C. Chisari, M. Bergamasco, and A. Frisoli, "A full upper limb robotic exoskeleton for reaching and grasping rehabilitation triggered by motor imagery-brain computer interface," in Rehabilitation Robotics (ICORR), 2015 IEEE International Conference on. IEEE, 2015, pp. 49-54.

[5] G. Pfurtscheller, G. Muller-Putz, R. Scherer, and C. Neuper, "Rehabilitation with brain-computer interface systems," Computer, 2008.

[6] N. Mrachacz-Kersting, N. Jiang, A. Stevenson, I. Niazi, V. Kostic, A. Pavlovic, S. Radovanovic, M. Djuric-Jovicic, F. Agosta, K. Dremstrup, and D. Farina, "Efficient neuroplasticity induction in chronic stroke patients by an associative braincomputer interface," Journal of neurophysiology, vol. 115, no. 3, pp. 1410-1421, 2016.

[7] K. K. Ang, C. Guan, K. Sui Geok Chua, B. T. Ang, C. Kuah, C. Wang, K. S. Phua, Z. Y. Chin, and H. Zhang, "Clinical study of neurorehabilitation in stroke using eeg-based motor imagery brain-computer interface with robotic feedback," in Engineering in Medicine and Biology Society (EMBC), 2010 Annual International IEEE Conference, 2010.

[8] K. K. Ang, C. Guan, K. S. G. Chua, B. T. Ang, C. W. K. Kuah, C. Wang, K. S. Phua, Z. Y. Chin, and H. Zhang, "A large clinical study on the ability of stroke patients to use an eegbased motor imagery brain-computer interface," Clinical EEG and Neuroscience, vol. 42, no. 4, pp. 253-258, 2011.

[9] A. Ramos-Murguialday, D. Broetz, M. Rea, L. Läer, Ö. Yilmaz, F. L. Brasil, G. Liberati, M. R. Curado, E. Garcia-Cossio, A. Vyziotis et al., "Brain-machine interface in chronic stroke rehabilitation: a controlled study," Annals of neurology, vol. 74, no. 1, 2013.

[10] B. M. Young, Z. Nigogosyan, A. Remsik, L. Walton, J. Song, V. A. Nair, S. W. Grogan, M. E. Tyler, D. F. Edwards, and K. Caldera, "Changes in functional connectivity correlate with behavioral gains in stroke patients after therapy using a braincomputer interface device," Frontiers in neuroengineering, vol. 7, 2014.

[11] T. Ono, K. Shindo, K. Kawashima, N. Ota, M. Ito, T. Ota, M. Mukaino, T. Fujiwara, A. Kimura, M. Liu et al., "Braincomputer interface with somatosensory feedback improves functional recovery from severe hemiplegia due to chronic stroke," Interaction of BCI with the underlying neurological conditions in patients: pros and cons, 2015. 
[12] A. Frisoli, C. Loconsole, D. Leonardis, F. Banno, M. Barsotti, C. Chisari, and M. Bergamasco, "A new gaze-bci-driven control of an upper limb exoskeleton for rehabilitation in realworld tasks," Systems, Man, and Cybernetics, Part C: Applications and Reviews, IEEE Transactions on, vol. 42, no. 6, pp. 1169-1179, 2012.

[13] S. Silvoni, A. Ramos-Murguialday, M. Cavinato, C. Volpato, G. Cisotto, A. Turolla, F. Piccione, and N. Birbaumer, "Braincomputer interface in stroke: a review of progress," Clinical EEG and Neuroscience, vol. 42, no. 4, pp. 245-252, 2011.

[14] S. de Vries, M. Tepper, B. Otten, and T. Mulder, "Recovery of motor imagery ability in stroke patients," Rehabilitation research and practice, vol. 2011, 2011.

[15] K. K. Ang and C. Guan, "Brain-computer interface in stroke rehabilitation," Journal of Computing Science and Engineering, vol. 7, no. 2, pp. 139-146, 2013.

[16] O. Bai, D. Huang, D.-Y. Fei, and R. Kunz, "Effect of realtime cortical feedback in motor imagery-based mental practice training," NeuroRehabilitation, vol. 34, no. 2, pp. 355-363, 2014.

[17] A. S. Royer, A. J. Doud, M. L. Rose, and B. He, "Eeg control of a virtual helicopter in 3-dimensional space using intelligent control strategies," Neural Systems and Rehabilitation Engineering, IEEE Transactions on, vol. 18, no. 6, pp. 581-589, 2010.

[18] G. Pfurtscheller and F. Lopes da Silva, "Event-related eeg/meg synchronization and desynchronization: basic," neurophysiology, vol. 110, no. 11, pp. 1842-1857, 1999.

[19] C. Guger, G. Edlinger, W. Harkam, I. Niedermayer, and G. Pfurtscheller, "How many people are able to operate an eeg-based brain- interface (bci)?" and Rehabilitation Engineering, IEEE Transactions on, vol. 11, no. 2, pp. 145-147, 2003.

[20] A. Vuckovic, "Motor questionnaire as a method to detect bci," in in Biomedical and Communication Technologies (ISABEL), 2010 3rd International Symposium on. IEEE, 2010, pp. 1-5.

[21] A. Ramos-Murguialday, M. Schürholz, V. Caggiano, M. Wildgruber, A. Caria, E. Hammer, S. Halder, and N. Birbaumer, "Proprioceptive feedback and brain computer interface based neuroprostheses," PloS one, vol. 7, no. 10, p. e47048, 2012.

[22] A. Chatterjee, V. Aggarwal, A. Ramos, S. Acharya, and N. V. Thakor, "Journal of neuroengineering and rehabilitation," Journal of NeuroEngineering and Rehabilitation, vol. 4, p. 40, 2007.

[23] T. Ono, K. Shindo, K. Kawashima, N. Ota, M. Ito, T. Ota, M. Mukaino, T. Fujiwara, A. Kimura, M. Liu et al., "Braincomputer interface with somatosensory feedback improves functional recovery from severe hemiplegia due to chronic stroke," Frontiers in neuroengineering, vol. 7, 2014.

[24] L. Yao, J. Meng, D. Zhang, X. Sheng, and X. Zhu, "Selective sensation based brain-computer interface via mechanical vibrotactile stimulation," Plos ONE, 2013.

[25] A. Gallace and C. Spence, "Touch and the body: The role of the somatosensory cortex in tactile awareness," Psyche: An Interdisciplinary Journal of Research on Consciousness, 2010.

[26] G. M. Goodwin, D. McCloskey, and P. Matthews, "The contribution of muscle afferents to keslesthesia shown by vibration induced illusions of movement and by the effects of paralysing joint afferents," Brain, vol. 95, no. 4, pp. 705-748, 1972.

[27] E. Naito, H. Ehrsson, S. Geyer, K. Zilles, and P. Roland, "Illusory arm movements activate cortical motor areas: a positron emission tomography study," The Journal of neuroscience, vol. 19, no. 14, pp. 6134-6144, 1999.

[28] R. Seitz and P. E. Roland, "Vibratory stimulation increases and decreases the regional cerebral blood flow and oxidative metabolism: a positron emission tomography (pet) study," Acta neurologica scandinavica, vol. 86, no. 1, pp. 60-67, 2009.

[29] E. Ribot-Ciscar, S. Tréfouret, J.-M. Aimonetti, S. Attarian, J. Pouget, and J.-P. Roll, "Is muscle spindle proprioceptive function spared in muscular dystrophies? a muscle tendon vibration study," Muscle \& nerve, vol. 29, no. 6, pp. 861-866, 2004.

[30] E. Ribot-Ciscar, M.-D. Bovis, J.-M. Aimonetti, B. Lapeyssonnie, E. Campana-Salort, J. Pouget, and S. Attarian, "Functional impact of vibratory proprioceptive assistance in patients with facioscapulohumeral muscular dystrophy," Muscle \& nerve, 2015.

[31] D. Leonardis, A. Frisoli, M. Barsotti, M. Carrozzino, and M. Bergamasco, "Multisensory feedback can enhance embodiment within an enriched virtual walking scenario," PRES-
ENCE: Teleoperators and Virtual Environments, vol. 23, no. 3, pp. 253-266, 2014.

[32] M. D. Rinderknecht, Y. Kim, L. Santos-Carreras, H. Bleuler, and R. Gassert, "Combined tendon vibration and virtual reality for post-stroke hand rehabilitation," in World Haptics Conference (WHC), 2013. IEEE, 2013, pp. 277-282.

[33] J. S. Schofield, M. R. Dawson, J. P. Carey, and J. S. Hebert, "Characterizing the effects of amplitude, frequency and limb position on vibration induced movement illusions: Implications in sensory-motor rehabilitation." Technology and health care: official journal of the European Society for Engineering and Medicine, vol. 23, no. 2, pp. 129-141, 2015.

[34] L. Yao, J. Meng, X. Sheng, D. Zhang, and X. Zhu, "A novel calibration and task guidance framework for motor imagery bci via a tendon vibration induced sensation with kinesthesia illusion," Journal of neural engineering, vol. 12, no. 1, 2015.

[35] D. Leonardis, A. Frisoli, M. Solazzi, and M. Bergamasco, "Illusory perception of arm movement induced by visuoproprioceptive sensory stimulation and controlled by motor imagery," in Haptics Symposium (HAPTICS), 2012 IEEE. IEEE, 2012, pp. 421-424.

[36] E. Tidoni, G. Fusco, D. Leonardis, A. Frisoli, M. Bergamasco, and S. M. Aglioti, "Illusory movements induced by tendon vibration in right-and left-handed people," Experimental brain research, vol. 233, no. 2, pp. 375-383, 2015.

[37] H. Ramoser, J. Muller-Gerking, and G. Pfurtscheller, “Optimal spatial filtering of single trial eeg during imagined hand movement," Rehabilitation Engineering, IEEE Transactions on, vol. 8, no. 4, pp. 441-446, 2000.

[38] G. Pfurtscheller, C. Neuper, C. Guger, W. Harkam, H. Ramoser, A. Schlogl, B. Obermaier, M. Pregenzer et al., "Current trends in graz brain-computer interface (bci) research," IEEE Transactions on Rehabilitation Engineering, vol. 8, no. 2, pp. 216-219, 2000.

[39] C. Guger, H. Ramoser, and G. Pfurtscheller, "Real-time eeg analysis with subject-specific spatial patterns for a braincomputer interface (bci)," Rehabilitation Engineering, IEEE Transactions on, vol. 8, no. 4, pp. 447-456, 2000.

[40] D. Leonardis, A. Frisoli, M. Barsotti, N. Vanello, and M. Bergamasco, "A comparison of algorithms for motor imagery for bci under different sensory feedback conditions," in Biomedical Robotics and Biomechatronics (BioRob), 2012 4th IEEE RAS E EMBS International Conference on. IEEE, 2012, pp. 1010-1015.

[41] A. Delorme and S. Makeig, "Eeglab: an open source toolbox for analysis of single-trial eeg dynamics including independent component analysis," Journal of neuroscience methods, vol. 134, no. 1, pp. 9-21, 2004.

[42] R. Grandchamp and A. Delorme, "Single-trial normalization for event-related spectral decomposition reduces sensitivity to noisy trials," Frontiers in Psychology, vol. 2, 2011.

[43] G. R. Müller-Putz, R. Scherer, C. Brunner, R. Leeb, and G. Pfurtscheller, "Better than random? a closer look on bci results," International Journal of Bioelectromagnetism, 2008.

[44] E. Naito, T. Kochiyama, R. Kitada, S. Nakamura, M. Matsumura, Y. Yonekura, and N. Sadato, "Internally simulated movement sensations during motor imagery activate cortical motor areas and the cerebellum," The Journal of neuroscience, vol. 22, no. 9, pp. 3683-3691, 2002.

[45] A. Forner-Cordero, M. Steyvers, O. Levin, K. Alaerts, and S. P. Swinnen, "Changes in corticomotor excitability following prolonged muscle tendon vibration," Behavioural brain research, vol. 190, no. 1, pp. 41-49, 2008.

[46] L. Casini, P. Romaiguère, A. Ducorps, D. Schwartz, J.-L. Anton, and J.-P. Roll, "Cortical correlates of illusory hand movement perception in humans: a meg study," Brain research, vol. 1121, no. 1, pp. 200-206, 2006. 